\title{
Article \\ Perioperative Chemotherapy with FLOT Scheme in Resectable Gastric Adenocarcinoma: A Preliminary Correlation between TRG and Radiomics
}

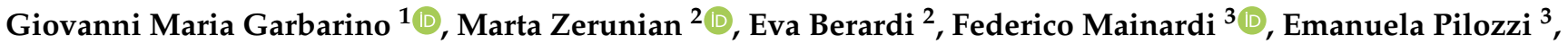 \\ Michela Polici ${ }^{2}(D)$, Gisella Guido ${ }^{2}$,, Carlotta Rucci ${ }^{2}$, Tiziano Polidori ${ }^{2}$, Mariarita Tarallo ${ }^{4}$, \\ Giovanni Guglielmo Laracca ${ }^{1}(0)$, Elsa Iannicelli ${ }^{2}$, Paolo Mercantini ${ }^{1}$, Bruno Annibale ${ }^{5}{ }^{\circledR}$, Andrea Laghi ${ }^{2}(\mathbb{D}$ \\ and Damiano Caruso ${ }^{2, *(1)}$
}

\section{check for} updates

Citation: Garbarino, G.M.; Zerunian, M.; Berardi, E.; Mainardi, F.; Pilozzi, E.; Polici, M.; Guido, G.; Rucci, C.; Polidori, T.; Tarallo, M.; et al. Perioperative Chemotherapy with FLOT Scheme in Resectable Gastric Adenocarcinoma: A Preliminary Correlation between TRG and Radiomics. Appl. Sci. 2021, 11, 9211. https://doi.org/10.3390/app11199211

Academic Editor: Francesco Bianconi

Received: 4 September 2021

Accepted: 24 September 2021

Published: 3 October 2021

Publisher's Note: MDPI stays neutral with regard to jurisdictional claims in published maps and institutional affiliations.

Copyright: (c) 2021 by the authors. Licensee MDPI, Basel, Switzerland. This article is an open access article distributed under the terms and conditions of the Creative Commons Attribution (CC BY) license (https:/ / creativecommons.org/licenses/by/ $4.0 /)$.
1 Gastrointestinal Surgery Unit, Department of Medical-Surgical Sciences and Translational Medicine, Sapienza University of Rome, Sant'Andrea Hospital, Via di Grottarossa 1035-39, 00189 Rome, Italy; giovannimaria.garbarino@uniroma1.it (G.M.G.); giovanniguglielmo.laracca@uniroma1.it (G.G.L.); paolo.mercantini@uniroma1.it (P.M.)

2 Radiology Unit, Department of Medical-Surgical Sciences and Translational Medicine, Sapienza University of Rome, Sant'Andrea Hospital, Via di Grottarossa 1035-39, 00189 Rome, Italy; marta.zerunian@uniroma1.it (M.Z.); eva.berardi@uniroma1.it (E.B.); michela.polici@uniroma1.it (M.P.); gisella.guido@uniroma1.it (G.G.); carlotta.rucci@uniroma1.it (C.R.); tiziano.polidori@uniroma1.it (T.P.); elsa.iannicelli@uniroma1.it (E.I.); andrea.laghi@uniroma1.it (A.L.)

3 Pathology Unit, Department of Clinical and Molecular Medicine, Sapienza University of Rome, Sant'Andrea Hospital, Via di Grottarossa 1035-39, 00189 Rome, Italy; federico.mainardi@uniroma1.it (F.M.); emanuela.pilozzi@uniroma1.it (E.P.)

4 Department of Surgery "Pietro Valdoni", Sapienza University of Rome, Policlinico Umberto I Hospital, Via Giovanni Maria Lancisi, 2, 00161 Roma, Italy; mariarita.tarallo@uniroma1.it

5 Gastroenterology Unit, Department of Medical Surgical Sciences and Translational Medicine, Sapienza University of Rome, Sant'Andrea Hospital, Via di Grottarossa 1035-39, 00189 Rome, Italy; bruno.annibale@uniroma1.it

* Correspondence: damiano.caruso@uniroma1.it; Tel.: +39-06-3377-5691

Featured Application: Radiomics may be a useful non-invasive biomarker in the assessment of response to perioperative chemotherapy in gastric cancer patients.

Abstract: Perioperative chemotherapy ( $\mathrm{p}-\mathrm{ChT}$ ) with a fluorouracil plus leucovorin, oxaliplatin, and docetaxel (FLOT) scheme is the gold standard of care for locally advanced gastric cancer. We aimed to test $\mathrm{CT}$ radiomics performance in early response prediction for $\mathrm{p}$-ChT. Patients with advanced gastric cancer who underwent contrast enhanced CT prior to and post $\mathrm{p}$-ChT were retrospectively enrolled. Histologic evaluation of resected specimens was used as the reference standard, and patients were divided into responders (TRG 1a-1b) and non-responders (TRG 2-3) according to their Becker tumor regression grade (TRG). A volumetric region of interest including the whole tumor tissue was drawn from a CT portal-venous phase before and after p-ChT; 120 radiomic features, both first and second order, were extracted. CT radiomics performances were derived from baseline $\mathrm{CT}$ radiomics alone and $\Delta$ Radiomics to predict response to $\mathrm{p}-\mathrm{ChT}$ according to the TRG and tested using a receiver operating characteristic (ROC) curve. The final population comprised 15 patients, $6(40 \%)$ responders and $9(60 \%)$ non-responders. Among pre-treatment CT radiomics parameters, Shape, GLCM, First order, and NGTDM features showed a significant ability to discriminate between responders and non-responders $(p<0.011)$, with Cluster Shade and Autocorrelation (GLCM features) having $\mathrm{AUC}=0.907 . \Delta$ Radiomics showed significant differences for Shape, GLRLM, GLSZM, and NGTDM features ( $p$ 0.007). MeshVolume (Shape feature) and LongRunEmphasis (GLRLM feature) had AUC $=0.889$. In conclusion, $C T$ radiomics may represent an important supportive approach for the radiologic evaluation of advanced gastric cancer patients.

Keywords: radiomics; gastric cancer; perioperative chemotherapy; response to treatment 


\section{Introduction}

Gastric cancer is one of the most common malignant tumors, and it ranks third worldwide in terms of mortality rates. Recently, with the increased progress in treatment approaches, patients with pathological stage I disease have achieved a cure rate of $90 \%$; nevertheless, the prognosis of advanced gastric cancer is still poor in Europe, and the 5 -year survival rate is reported to be about 25\% [1,2]. Radical gastrectomy with D2 lymphadenectomy has historically been the milestone treatment for locally advanced gastric cancer (Stage IB-III) [3,4]. Since the publication of the MAGIC trial results showing that three preoperative and three postoperative cycles of epirubicin, cisplatin, and fluorouracil (ECF) improves progression-free and overall survival, the use of perioperative chemotherapy (p-ChT) has spread throughout Western countries [5]. Currently in Europe, the gold standard treatment for patients with locally advanced gastric cancer is radical gastrectomy with D2 lymphadenectomy and p-ChT with fluorouracil plus leucovorin, oxaliplatin, and docetaxel (FLOT) [6].

The present trial compared the perioperative docetaxel-based triplet FLOT with an anthracycline-based triplet of either ECF or ECX (epirubicin, cisplatin, and capecitabine) for patients with resectable gastric or esophagogastric junction adenocarcinoma. The results of the trial showed that the patients treated with FLOT achieved a significantly higher rate of pathological complete regression $(16 \%$ vs. $6 \% ; p=0.02)$ associated with an improved overall survival with an estimated advantage of 15 months (50 vs. 35 months; $p=0.012$ ) when compared to the ECF/ECX group. However, not all patients are responders who can benefit from this approach. In non-responder patients, costly and ineffective preoperative chemotherapy could be avoided if they are assessed beforehand [7-9]. Currently, the only objective method of determining whether a patient has responded to preoperative chemotherapy is the evaluation of the tumor regression grade (TRG) of a surgical specimen. In order to exclude non-responder patients from preoperative chemotherapy, a diagnostic method to discriminate responder and non-responder patients should be identified at the time of the diagnosis.

Radiomics is a tool able to extract ultrastructural quantitative data from previously acquired medical images (e.g., computed tomography, magnetic resonance imaging, ultrasound), providing features that characterize the spatial relationships of signal intensities in a specific tissue (e.g., a tumoral lesion) [10]. Radiomics has already demonstrated its usefulness in predicting treatment response across a range of cancer types and imaging modalities [10-15]. Regarding gastric cancer, CT radiomics has already been shown to be a promising preoperative non-invasive prognostic biomarker [16]. Pre-treatment radiomics extracted from a baseline CT examination could also be used to provide important information about the response rate to preoperative chemotherapy for gastric cancer. This would improve patients' chances of selection for multimodal treatment $[17,18]$. Therefore, radiomics may represent an innovative non-invasive biomarker for the response to perioperative chemotherapy in locally advanced gastric cancer. Thus, the aim of this study is to assess the performance of $\mathrm{CT}$ radiomics in predicting the response to perioperative chemotherapy with a FLOT regimen in gastric cancer patients.

\section{Materials and Methods}

\subsection{Study Population}

The current retrospective observational study regards all patients undergoing surgery for gastric cancer at the Gastrointestinal Surgery Unit of Sant'Andrea University Hospital between January 2019 and November 2020. The study was conducted in accordance with the Declaration of Helsinki and its later amendments. Formal Institutional Review Board approval was not required due to the non-interventional, retrospective nature of the study. All patients provided signed consent for data treatment and analysis for scientific purposes before any procedures.

Data were registered in a prospectively maintained database of all patients undergoing gastric resection with perioperative chemotherapy for gastric cancer. The following 
data were included in the study: demographics (age, sex, ASA score, BMI, comorbidities), characteristics of the tumoral lesion and its pathology, and baseline and post-perioperative chemotherapy CT examinations. The following inclusion criteria were considered: (1) having previously undergone a gastrectomy with a histologically proven diagnosis of gastric carcinoma with baseline staging CT scan pre- and post-perioperative chemotherapy and a multidisciplinary team evaluation; (2) preoperative tumor stage as follows: cT2-T4a, cN0-N3, or M0; (3) age > 18 years old; and (4) having undergone perioperative chemotherapy with docetaxel, oxaliplatin, leucovorin, and 5-fluorouracil (FLOT). The exclusion criteria were: (1) unavailability of both baseline and post-perioperative chemotherapy CT examinations; (2) disease progression (metastatic tumoral spread) during perioperative chemotherapy not eligible for further surgery; and (3) the use of a different chemotherapy scheme than FLOT.

Patients were divided into two groups following the Becker TRG classification system: non-responders (TRG 1a-1b) and responders (TRG 2-3) [19-21]. Tumor staging was evaluated according to the American Joint Committee on Cancer (AJCC) Staging System, 8th edition [20]. All patients underwent a total body CT scan and endoscopic ultrasound for preoperative clinical staging, and the clinical pathway was determined by the multidisciplinary team (MDT).

\subsection{CT Acquisition Protocol}

Each patient with a histological diagnosis of gastric cancer underwent a pre- and post-p-ChT total body CT scan with contrast medium injection. CT scans were acquired on 128-slice CT (GE Revolution EVO Slice CT Scanner, GE Medical Systems, Milwaukee, WI, USA) in supine position, at end-inspiration and with cranio-caudal scanning. For the unenhanced imaging, arterial and delayed phase Z-axis coverage included the diaphragm apex to the iliac bone, and the portal-venous phase was scanned from the sovraclavear space to the pubic symphysis. For the purposes of this study, only the portal-venous phase was assessed.

All patients underwent gastric lumen distention by drinking 4 glasses $(125 \mathrm{~mL})$ of water directly before the $\mathrm{CT}$ image acquisition. Contrast medium intravenous injection was tailored for each patient according to lean body weight (LBW): each patient received $0.7 \mathrm{gI} / \mathrm{kg}$ LBW $[22,23]$ and the result divided by contrast medium concentration $(\mathrm{mgI} / \mathrm{mL})$ obtaining the volume of administration. Iso-osmolar non-ionic contrast medium (iodixanol $320 \mathrm{mgI} / \mathrm{mL}$, Visipaque 320; GE Healthcare, Cork, Ireland) was administered through an 18-20 gauge antecubital intravenous access, followed by $40 \mathrm{~mL}$ of saline solution with a contrast media injection system (Medrad ${ }^{\circledR}$ Centargo, Bayer) with a flow rate of $3 \mathrm{~mL} / \mathrm{s}$. Post-contrast CT scan timing was based on the bolus-tracking method (Smart Prep, GE, Milwaukee, WI, USA) by placing a $150 \mathrm{HU}$-threshold region of interest (ROI) within the lumen of the abdominal aorta at the celiac tripod level. The late arterial phase ( $18 \mathrm{~s}$ after threshold reached), portal-venous phase (70 s after the threshold reached), and delayed phase (180 s after threshold reached) were performed for each patient's unenhanced phase.

CT technical parameters were: tube voltage $100 \mathrm{kV}$; tube current modulation was applied by using SMART mA (GE Healthcare, Milwaukee, WI, USA) ranging from 130 to $300 \mathrm{mAs}$; spiral pitch factor 0.98 ; section collimation $64 \times 0.625 \mathrm{~mm}$; rotation time $0.6 \mathrm{~s}$. All CT images were reconstructed with slice thickness of $1.25 \mathrm{~mm}$ with standard soft tissue reconstruction, by applying iterative reconstruction at 50\% (ASiR-V, GE Healthcare, Milwaukee, WI, USA). No other iterative reconstruction was analyzed [24].

\subsection{Image Segmentation Analysis}

CT examinations were retrospectively analyzed in consensus by two radiologists (EB and DC, with 5 and 10 years of experience in abdominal oncology, respectively). For each patient, both the baseline and post-p-ChT CT scans were analyzed by volumetric tumor segmentation using 3D Slicer software (version 4.10.2, http: / / www.slicer.org, accessed on 24 October 2020) that had already been tested on gastric neoplasms $[17,25]$. The volumetric 
region of interest (VOI) was manually outlined slice-by-slice on portal-venous phase contrast-enhanced CT examinations to cover the entire gastric cancer area while avoiding the inclusion of surrounding healthy mucosa, gas, and perigastric fat in the analysis.

\subsection{Radiomics Features Extraction}

Radiomics features were extracted from the VOIs using a dedicated 3D Slicer radiomics extension (pyradiomics library, [26]), as shown in Figure 1. No spatial filter alteration scaling was applied to the radiomic features. In particular, several radiomics features of the first and second order were extracted as follows: (a) first-order statistics (19 features), (b) 2D and 3D shape-based features (26 features), (c) gray level co-occurrence matrix (GLCM, 24 features), (d) gray level run length matrix (GLRLM, 16 features), (e) gray level size zone matrix (GLSZM, 16 features), (f) neighboring gray tone difference matrix (NGTDM, 5 features), and (g) gray level dependence matrix (GLDM, 14 features). From baseline and post-pChT CT images, a total of 120 radiomic features were extracted. Additionally, a $\Delta$ Radiomics number was obtained using the following formula:

$$
\Delta \text { Radiomics }=\text { Radiomics Post-CHT }- \text { Radiomics Post-CHT }
$$
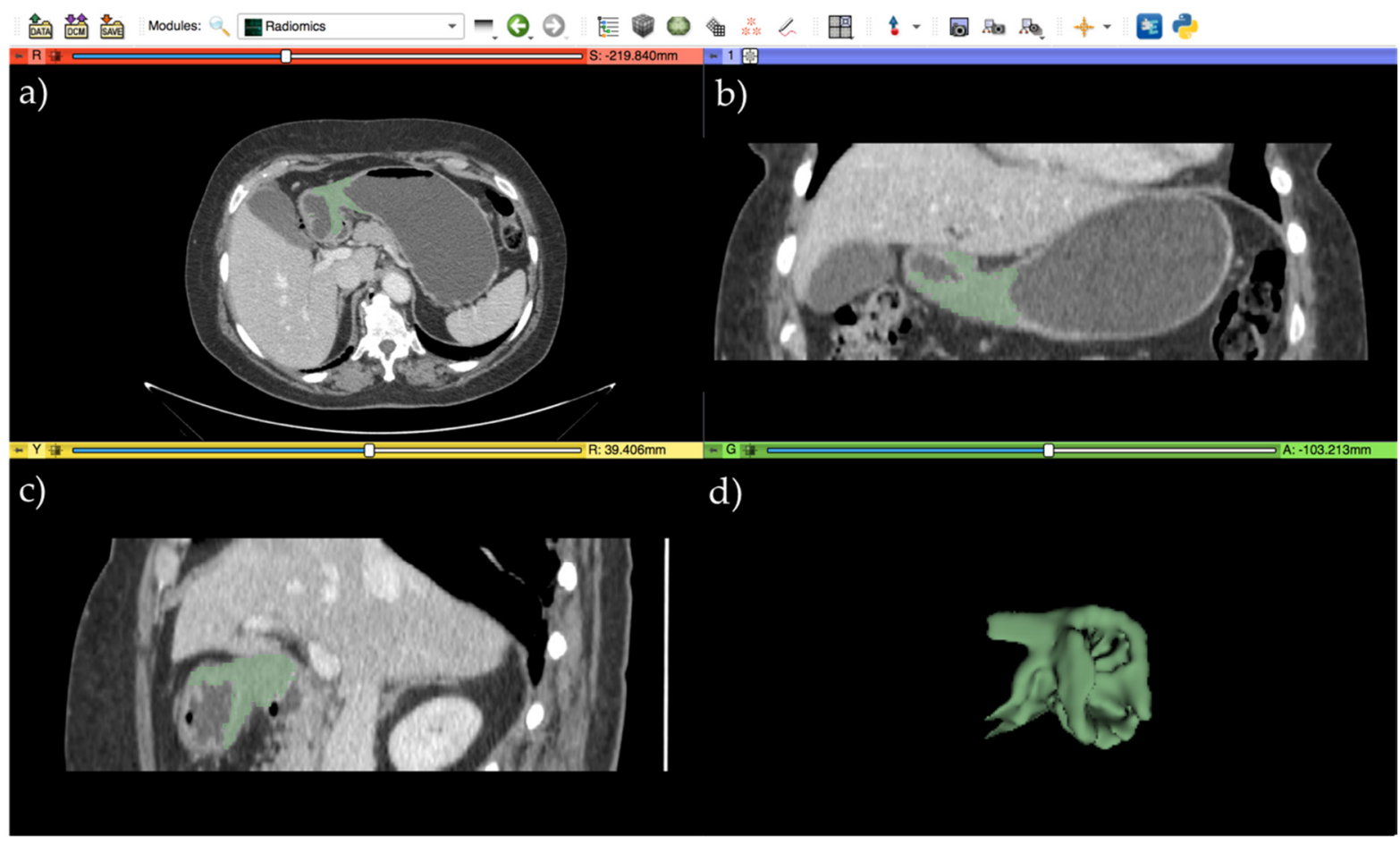

Figure 1. A volumetric manual segmentation performed with 3D Slicer software (version 4.10.2, http:/ / www.slicer.org (accessed on 24 October 2020)) on a portal-venous phase CT scan of a 78-year-old woman with locally advanced gastric cancer after perioperative chemotherapy. (a-c) Axial, coronal, and sagittal plane views respectively. (d) The final 3D representation of the whole tumor segment, avoiding the surrounding healthy mucosa, gas, and perigastric fat.

\subsection{Statistical Analysis}

Continuous data were expressed as the mean \pm standard deviation. Differences in continuous parametric variables were compared using the unpaired Student's $t$ test, and the Mann-Whitney $U$ test was used for the continuous nonparametric variables. The categorical variables were expressed by numbers and percentages and their comparisons were calculated with the $\chi^{2}$ test or Fisher's exact test with or without Yates correction. Our hypothesis test for all the comparisons performed considered $\mathrm{H}_{0}$ as no differences between responders and non-responders while $\mathrm{H}_{1}$ represented the presence of differences between the two groups. 
The diagnostic performance of radiomic features extracted from baseline CT scans to differentiate responder from non-responder patients was assessed using the receiver operating curve (ROC), calculating area under the curve (AUC), sensitivity, specificity, and accuracy. The diagnostic performance of $\triangle$ Radiomics was assessed with ROC curve analysis. Significance was defined as $p<0.05$. Statistical analysis was performed using the SPSS 25.0 (SPSS, Inc., Chicago, IL, USA) and MedCalc software (MedCalc Software, version15, Ostend, Belgium).

\section{Results}

\subsection{Study Population}

Between January 2019 and November 2020, a total of 48 patients underwent a gastrectomy with perioperative chemotherapy for gastric adenocarcinoma at our institution. Twenty-seven patients who underwent chemotherapy regimens other than FLOT, two patients without available pre- and post-perioperative chemotherapy CT scans, and four patients who had their radiological disease progression assessed with CT scans after perioperative FLOT therapy were excluded from the study. Fifteen patients fulfilling the study criteria were included in the study. Patients were divided into two groups: $9(60 \%)$ non-responders to p-ChT (TRG1a-1b) and 6 (40\%) responders to p-ChT (TRG2-3) (Figure 2).

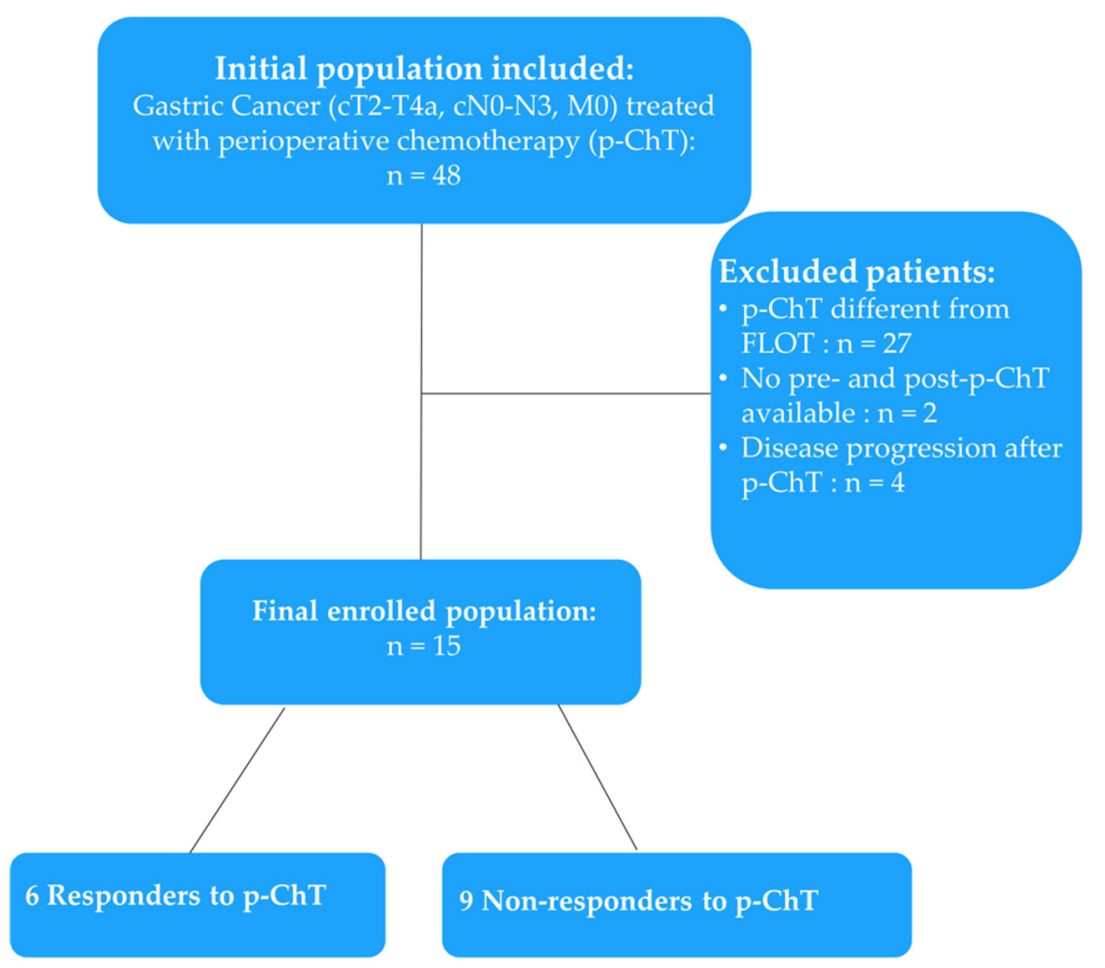

Figure 2. Enrollment population flowchart with inclusion and exclusion criteria and the final enrolled population.

\subsection{Demographic Characteristics}

No significant differences were observed between the two groups concerning the demographic characteristics age, sex, body mass index (BMI), American Society of Anesthesiology (ASA) score, comorbidities, and tumor histotype and location (Table 1). A nonsignificant trend of larger tumors was observed in the non-responder group $(2.2 \pm 0.8 \mathrm{~cm}$ vs. $3.4 \pm 1.1 \mathrm{~cm} ; p=0.073)$. Ten patients underwent subtotal gastrectomy $(30 \% ; p=0.264)$ and 5 patients underwent a total gastrectomy $(60 \% ; p=0.264)$. Six patients were treated by open approach $(66.7 \% ; p=0.237)$ and nine patients by laparoscopy $(22.2 \% ; p=0.237)$. Postoperative morbidity occurred more frequently in the non-responder group (14.3\%; $p=0.170)$, and 30-day mortality was zero in both groups. 
Table 1. Baseline characteristics of the included patients.

\begin{tabular}{|c|c|c|c|}
\hline & $\begin{array}{l}\text { Responders } \\
n=6\end{array}$ & $\begin{array}{c}\text { Non-Responders } \\
n=9\end{array}$ & $p$ \\
\hline Age (years, mean $\pm S D$ ) & $59.9( \pm 12.6)$ & $64.8( \pm 15.2)$ & 0.224 \\
\hline Gender F/M & $3 / 3$ & $3 / 6$ & 0.519 \\
\hline BMI (mean, \pm SD) & $23.8( \pm 2.2)$ & $24.8( \pm 3.1)$ & 0.639 \\
\hline $\operatorname{ASA}(n, \%)$ & & & 0.174 \\
\hline 1 & $0(0.0 \%)$ & $1(11.1 \%)$ & \\
\hline 2 & $3(50.0 \%)$ & $2(22.2 \%)$ & \\
\hline 3 & $3(50.0 \%)$ & $6(66.6 \%)$ & \\
\hline 4 & $0(0.0 \%)$ & $0(0.0 \%)$ & \\
\hline Comorbidities (n, \%) & $3(50.0 \%)$ & $4(44.4 \%)$ & 0.408 \\
\hline Histotype & & & 0.274 \\
\hline Non poorly cohesive & $3(50.0 \%)$ & $6(66.6 \%)$ & \\
\hline Poorly cohesive & $2(33.3 \%)$ & $0(0.0 \%)$ & \\
\hline Poorly cohesive, signet-ring cell & $0(0.0 \%)$ & $1(11.1 \%)$ & \\
\hline Mixed & $1(16.7 \%)$ & $2(22.2 \%)$ & \\
\hline Tumor Location & & & 0.255 \\
\hline Cardias & $1(16.7 \%)$ & $0(0.0 \%)$ & \\
\hline Subcardial & $1(16.7 \%)$ & $1(11.1 \%)$ & \\
\hline Fundus & $0(0.0 \%)$ & $0(0.0 \%)$ & \\
\hline Body & $0(0.0 \%)$ & $1(11.1 \%)$ & \\
\hline Angulus & $2(33.3 \%)$ & $1(11.1 \%)$ & \\
\hline Antrum & $1(16.7 \%)$ & $6(66.7 \%)$ & \\
\hline Pylorus & $1(16.7 \%)$ & $0(0.0 \%)$ & \\
\hline Tumor size (cm, mean $\pm \mathrm{SD})$ & $2.2( \pm 0.8)$ & $3.4( \pm 1.1)$ & 0.073 \\
\hline
\end{tabular}

Body mass index (BMI), American Society of Anesthesiology (ASA).

\subsection{Pathological and Long-Term Oncological Outcomes}

Pathological examination of the resected specimens showed comparable $\mathrm{T}$ stages $(p=0.315), \mathrm{N}$ stages $(p=0.397)$, number of retrieved lymph nodes ( 26.0 vs. $24.2 ; p=1.000)$, and number of positive nodes between groups $(p=0.388)$. Furthermore, R0 resection was achieved in $100 \%$ of cases in both groups $(p=1.000)$. Finally, post-preoperative TNM stage was comparable between the groups $(p=0.774)$. Results are listed in Table 2.

Table 2. Oncological outcomes including ypTNM stage, resection margin, node details, and lymphovascular/perineural invasion in responder vs. non-responder patients.

\begin{tabular}{ccc}
\hline & $\begin{array}{c}\text { Responders } \\
\mathbf{n}=\mathbf{6}\end{array}$ & $\begin{array}{c}\text { Non- Responders } \\
n=9\end{array}$ \\
\hline T-stage (n, \%) & & \\
ypT1 & $3(50.0 \%)$ & $2(22.2 \%)$ \\
ypT2 & $1(16.7 \%)$ & $3(33.3 \%)$ \\
ypT3 & $1(16.7 \%)$ & $4(44.4 \%)$ \\
ypT4a & $1(16.7 \%)$ & $0(0.0 \%)$ \\
ypT4b & $0(0.0 \%)$ & $0(0.0 \%)$ \\
\hline N-stage (n, \%) & & \\
ypN0 & $5(83.3 \%)$ & $1(11.1 \%)$ \\
ypN1 & $0(0.0 \%)$ & $2(22.2 \%)$ \\
ypN2 & $0(0.0 \%)$ & $2(22.2 \%)$ \\
ypN3 & $1(16.7 \%)$ & \\
M-stage (n, \%) & & $9(100 \%)$ \\
ypM0 & $6(100 \%)$ & 0.397 \\
ypM1 & $0(0.0 \%)$ & \\
\hline
\end{tabular}


Table 2. Cont.

\begin{tabular}{|c|c|c|c|}
\hline & $\begin{array}{c}\text { Responders } \\
n=6\end{array}$ & $\begin{array}{c}\text { Non- Responders } \\
n=9\end{array}$ & $p$ \\
\hline $\mathrm{R} 0$ resection $(\mathrm{n}, \%)$ & $6(100 \%)$ & $9(100 \%)$ & 1.000 \\
\hline Retrieved nodes (mean $\pm S D$ ) & $26.0( \pm 10.5)$ & $24.2( \pm 7.3)$ & 1.000 \\
\hline Positive nodes (mean $\pm S D$ ) & $2.7( \pm 6.5)$ & $3.4( \pm 3.9)$ & 0.388 \\
\hline Node ratio (mean $\pm \mathrm{SD}$ ) & $0.1( \pm 0.3)$ & $0.1( \pm 0.1)$ & 0.388 \\
\hline Lymphovascular invasion $(\mathrm{n}, \%)$ & $2(33.3 \%)$ & $4(44.4 \%)$ & 1.000 \\
\hline Perineural invasion $(\mathrm{n}, \%)$ & $1(16.7 \%)$ & $3(33.3 \%)$ & 0.604 \\
\hline ypTNM stage (n, \%) & & & 0.774 \\
\hline yI & $3(50.0 \%)$ & $3(33.3 \%)$ & \\
\hline yII & $2(33.3 \%)$ & $4(44.4 \%)$ & \\
\hline yIII & $1(16.7 \%)$ & $1(11.1 \%)$ & \\
\hline yIV & $0(0.0 \%)$ & $1(11.1 \%)$ & \\
\hline
\end{tabular}

\subsection{Radiomics Features Extraction}

Radiomics features of pre-p-ChT CT examinations showed significant differences between responders and non-responders for Shape, GLCM, First order, and NGTDM features. In particular, the extracted radiomics parameters showed significant differences in LeastAxisLength (Shape feature; $p=0.017$ ), Cluster Shade and Autocorrelation (GLCM features; $p=0.007$ and 0.005, respectively), Skewness (First order feature; $p=0.012$ ), and Strength (NGTDM feature; $p=0.049$ ). The diagnostic performance of these features was tested with ROC curves showing AUCs of 0.815 for LeastAxisLength, AUC of 0.907 for both Cluster Shade and Autocorrelation, an AUC of 0.889 for Skewness, and an AUC of 0.815 for Strength (all $p<0.011$ ). Complete results are shown in Table 3 and Figure 3.

Table 3. Pre-p-ChT radiomics analysis between responders and non-responders expressed as simple comparison analysis (Student's $t$ test/Mann-Whitney $\mathrm{U}$ ) and the receiver operating characteristic (ROC) curve analysis with the area under the curve (AUC), sensitivity, specificity, and interclass coefficient (IC).

\begin{tabular}{|c|c|c|c|c|c|c|c|c|c|}
\hline \multirow{2}{*}{\multicolumn{2}{|c|}{ Features ( \pm SD) }} & \multicolumn{3}{|c|}{ Student's $t$ Test/Mann-Whitney $\mathrm{U}$} & \multicolumn{5}{|c|}{ ROC Curve Analysis } \\
\hline & & \multirow{2}{*}{$\begin{array}{c}\text { Responders } \\
40,710,033,491,504,200 \\
( \pm 10,571,464,896,142,900)\end{array}$} & \multirow{2}{*}{$\begin{array}{c}\text { Non-Responders } \\
25,790,429,154,707,500 \\
( \pm 10,255,032,505,952,900)\end{array}$} & \multirow{2}{*}{$\frac{p}{0.017}$} & \multirow{2}{*}{$\begin{array}{l}\text { AUC } \\
0.815\end{array}$} & \multirow{2}{*}{ 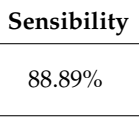 } & \multirow{2}{*}{$\frac{\text { Specificity }}{66.67 \%}$} & \multirow{2}{*}{$\begin{array}{c}\mathbf{9 5} \% \mathrm{CI} \\
0.53-0.96\end{array}$} & \multirow{2}{*}{$\begin{array}{c}p \\
0.011\end{array}$} \\
\hline Shape & LeastAxisLength & & & & & & & & \\
\hline \multirow{2}{*}{ GLCM } & Cluster Shade & $\begin{array}{c}0.386 \\
( \pm 0.268)\end{array}$ & $\begin{array}{c}146,046,470,600.51 \\
( \pm 221,971,036,175.30)\end{array}$ & 0.007 & 0.907 & $66.67 \%$ & $100 \%$ & $0.64-0.99$ & $<0.0001$ \\
\hline & Autocorrelation & $\begin{array}{c}600,512,843,926.50 \\
( \pm 266,967,395,651.26)\end{array}$ & $\begin{array}{c}172,336,715,286.11 \\
( \pm 225,325,689,588.11)\end{array}$ & 0.005 & 0.907 & $88.89 \%$ & $83.33 \%$ & $0.64-0.99$ & $<0.0001$ \\
\hline $\begin{array}{l}\text { First } \\
\text { order }\end{array}$ & Skewness & $\begin{array}{c}-41,087,393,947.19 \\
( \pm 92,868,722,144.65)\end{array}$ & $\begin{array}{c}-266,130,994,643.65 \\
( \pm 242,387,476,528.67)\end{array}$ & 0.012 & 0.889 & $88.89 \%$ & $83.33 \%$ & $0.62-0.99$ & $<0.0001$ \\
\hline NGTDM & Strength & $\begin{array}{c}0.099 \\
( \pm 0.065)\end{array}$ & $\begin{array}{c}12,828,807,550.54 \\
( \pm 38,486,422,650.55)\end{array}$ & 0.049 & 0.815 & $55.56 \%$ & $100 \%$ & $0.53-0.96$ & 0.007 \\
\hline
\end{tabular}

$\triangle$ Radiomics values showed significant differences between responders and nonresponders for Shape, GLRLM, GLSZM, and NGTDM features. Specific features include MeshVolume, LeastAxisLength, and SurfaceVolume (Shape features; $p=0.012,0.036$, and 0.020 respectively); LongRunEmphasis (GLRLM feature; $p=0.039$ ); LargeAreaLowGrayLevelEmphasis (GLSZM feature; $p=0.017$ ); and Contrast (NGTDM feature; $p=0.049$ ). Further, ROC curve analysis showed significant results for the abovementioned $\Delta$ Radiomics features with an AUC of 0.889 for MeshVolume, 0.833 for LeastAxisLength, 0.852 for SurfaceVolume, 0.889 for LongRunEmphasis, 0.833 for LargeAreaLowGrayLevelEmphasis, and 0.796 for Contrast (all $p<0.007$ ). Full results including sensitivity, specificity, and confidence interval (CI) are reported in Table 4 and Figure 4. 

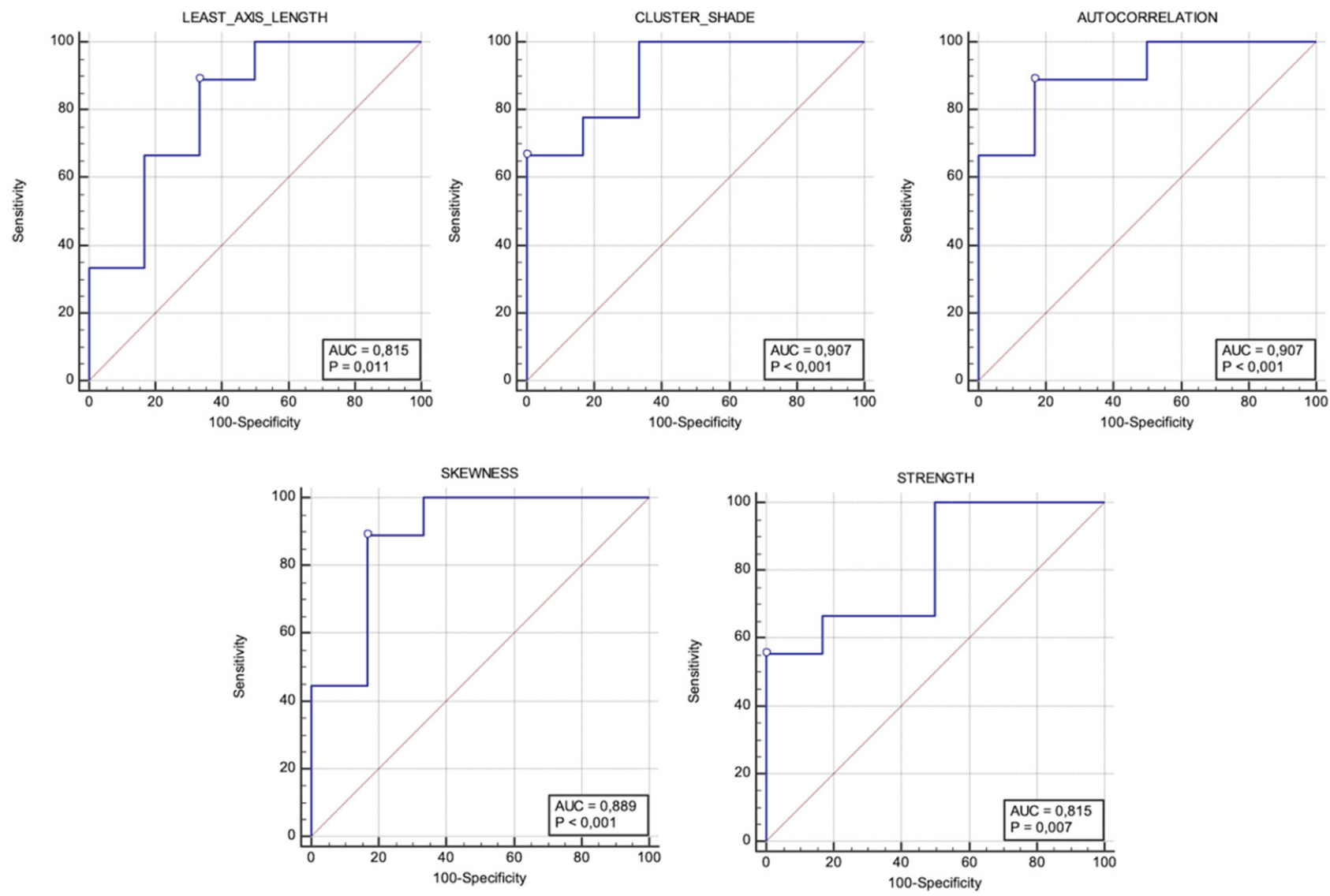

Figure 3. Significant receiver operating characteristic (ROC) curves of the radiomic features extracted from pre-perioperative chemotherapy CT scans. The area under the curve (AUC) and $p$-value are reported in each ROC curve. The best AUC achieved is represented by Cluster Shade and Autocorrelation.

Table 4. $\Delta$ Radiomics analysis between responders and non-responders expressed as a simple comparison analysis (Student's $\mathrm{t}$ test/Mann-Whitney $\mathrm{U}$ ) and receiver operating characteristic (ROC) curve analysis with the area under the curve (AUC), sensitivity, specificity, and 95\% confidence interval (95\%CI).

\begin{tabular}{|c|c|c|c|c|c|c|c|c|c|}
\hline \multirow{2}{*}{\multicolumn{2}{|c|}{ Features ( \pm SD) }} & \multicolumn{3}{|c|}{ Student's $t$ Test/Mann-Whitney $\mathrm{U}$} & \multicolumn{5}{|c|}{ ROC Curve Analysis } \\
\hline & & Responders & Non-Responders & $p$ & AUC & Sensitivity & Specificity & $95 \% \mathrm{CI}$ & $p$ \\
\hline \multirow{3}{*}{ Shape } & MeshVolume & $\begin{array}{c}47,726,724,383.33 \\
( \pm 116826556826.61)\end{array}$ & $\begin{array}{c}13,076,118.00 \\
( \pm 30921508.47)\end{array}$ & 0.012 & 0.889 & $66.67 \%$ & $100 \%$ & $0.62-0.99$ & $<0.0001$ \\
\hline & LeastAxisLength & $\begin{array}{l}12,285,531,715,430,500 \\
( \pm 14137871167552600)\end{array}$ & $\begin{array}{c}5,973,056,305,063,620 \\
( \pm 249,77,695,721,154,400)\end{array}$ & 0.036 & 0.833 & $77.78 \%$ & $100 \%$ & $0.55-0.97$ & 0.0045 \\
\hline & SurfaceVolume & $\begin{array}{c}0.014 \\
( \pm 0.05200651)\end{array}$ & $\begin{array}{c}0.1233 \\
( \pm 0.089998892)\end{array}$ & 0.020 & 0.852 & $88.89 \%$ & $83.33 \%$ & $0.57-0.97$ & 0.0021 \\
\hline GLRLM & LongRunEmphasis & $\begin{array}{c}2,579,499,151,841.67 \\
( \pm 3.283)\end{array}$ & $\begin{array}{l}149,429,893,302.00 \\
\quad( \pm 1.43237)\end{array}$ & 0.039 & 0.889 & $66.7 \%$ & $100 \%$ & $0.62-0.99$ & $<0.0001$ \\
\hline GLSZM & $\begin{array}{l}\text { LargeAreaLowGray- } \\
\text { LevelEmphasis }\end{array}$ & $\begin{array}{l}408,041,364,296.00 \\
\quad( \pm 3.38877)\end{array}$ & $\begin{array}{l}36,590,288,676.44 \\
\quad( \pm 1.9542)\end{array}$ & 0.017 & 0.833 & $100 \%$ & $66.67 \%$ & $0.55-0.97$ & 0.007 \\
\hline NGTDM & Contrast & $\begin{array}{c}0.00000 \\
( \pm 0.00571308)\end{array}$ & $\begin{array}{c}0.01000 \\
( \pm 0.00986065)\end{array}$ & 0.049 & 0.796 & $66.67 \%$ & $83.33 \%$ & $0.53-0.96$ & 0.005 \\
\hline
\end{tabular}



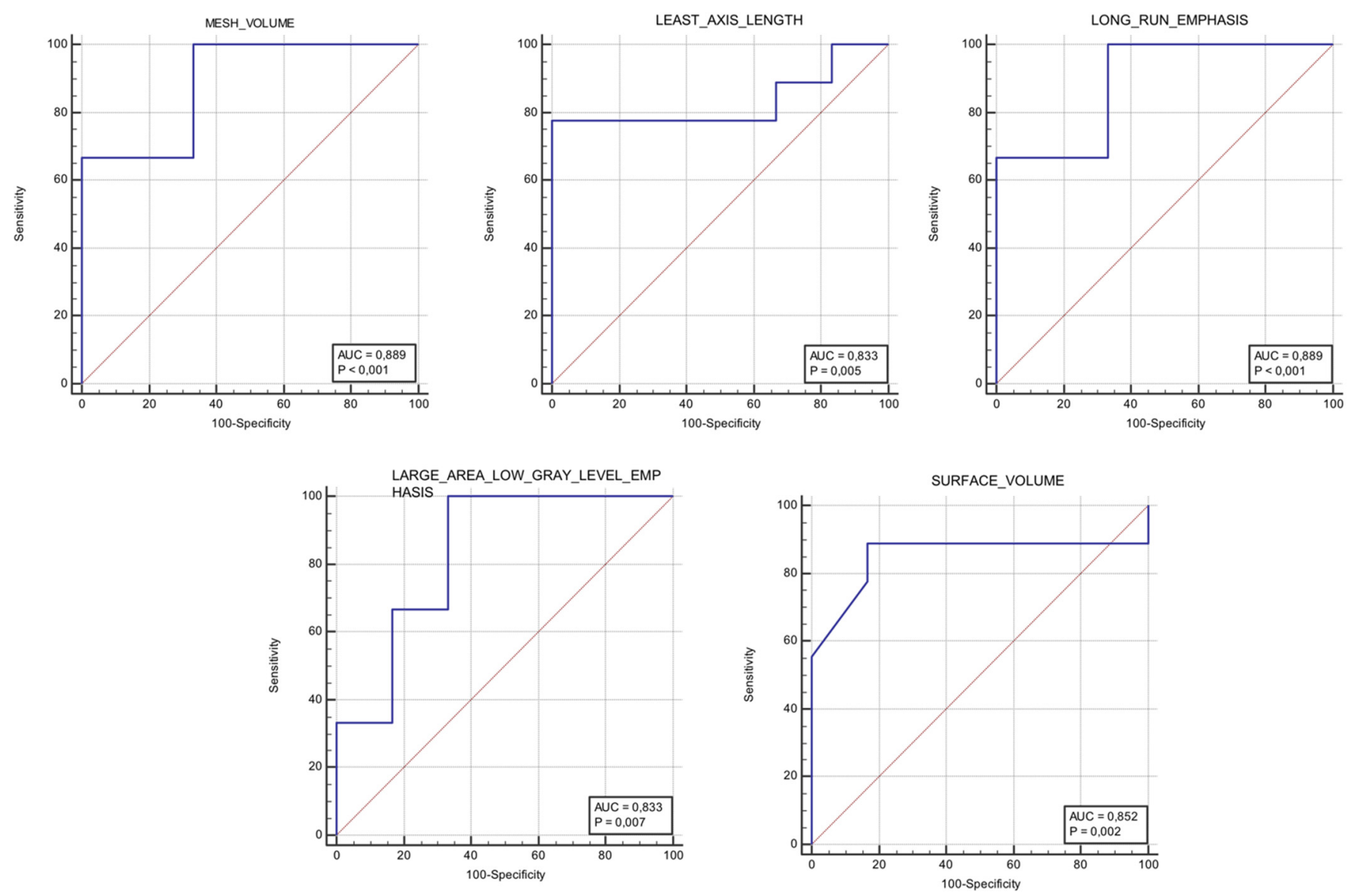

Figure 4. Significant receiver operating characteristic (ROC) curves of $\Delta$ Radiomics obtained from the differences between preperioperative chemotherapy CT and post-perioperative chemotherapy CT. The area under the curve (AUC) and the $p$-value are reported in each ROC curve. The best AUC achieved is represented by MeshVolume and LongRunEmphasis features.

\section{Discussion}

Our results showed significant performances from pre-p-ChT radiomics and $\Delta$ Radiomics in differentiating between responder and non-responder patients with gastric cancer treated with a FLOT regimen before surgery. The best performance for pre-p-ChT radiomics showed an AUC of 0.907 for Cluster, Shade, and Autocorrelation (GLCM features) with sensitivity of $66.67 \%$ and $88.89 \%$ and specificity of $100 \%$ and $83.33 \%$, respectively (all $p<0.0001$ ). Additionally, $\Delta$ Radiomics showed interesting results in terms of diagnostic performance with the best ROC curve showing an AUC $=0.889$ for MeshVolume (Shape feature) and for LongRunEmphasis (GLRLM feature), with sensitivity and specificity of $66.67 \%$ and $100 \%$ for both (all $p<0.0001$ ). Up to now, only a few studies have assessed the role of radiomics in the prediction of response to perioperative or neoadjuvant chemotherapy [17,27-29]. To the best of our knowledge, no CT radiomics studies predicting the response to perioperative chemotherapy with a FLOT regimen in gastric cancer patients have been performed.

The potential of radiomics in the early identification of perioperative treatment response represents a great opportunity for developing treatment strategies. Neoadjuvant chemotherapy responders showed an improvement in survival after gastrectomy [27]. The possibility of the early identification of non-responder patients would allow a differently tailored therapeutic strategy.

Different aspects of gastric cancer disease and radiomics have been investigated in several studies, which have included correlations with histological grade [28], staging, and therapeutic outcome [29-33]. As mentioned above, some studies have reported interesting results in response to neoadjuvant chemotherapy assessment. In particular, Giganti and colleagues performed pre-neoadjuvant $\mathrm{ChT}$ radiomics on 34 gastric cancer patients, cor- 
relating the extracted features with TRG. Their results indicated that three pre-treatment radiomic parameters (Entropy, Root Mean Square, and Range) have a possible predictive value in the discrimination between responders and non-responders in patients naïve for treatment.

Discrepancies with our study in terms of different significant radiomic features could be related to the small population sample and to their use of filtered radiomics that enhance some features more than others. Another interesting study performed by Li Z. et al. assessed the role of radiomics in the response to neoadjuvant chemotherapy prediction. The authors enrolled 30 patients and performed radiomics on pre-treatment CT on both arterial and portal-venous phases. Results showed how portal-venous phase radiomics performed better than arterial phase radiomics in the prediction of TRG, with an AUC of 0.72 compared to one of 0.60 [17]. These results are important in terms of radiomics application due to the increased availability of portal-venous phase in comparison to arterial phase in oncologic studies, and this reinforced our decision to test radiomics on the portal-venous phase, even though more data are needed for confirmation.

Moreover, Sun K-Y. et al. performed a combined multivariate analysis on 106 patients divided into training and validation cohorts. The researchers proposed a RAD score selected by the randomized decision tree method, and then integrated it into a RAD clinical score. Results showed how RAD score alone showed the best performance in assessing response to neoadjuvant $\mathrm{ChT}$ compared to RAD clinical score and clinical score only (AUC 0.82 vs. 0.70 and 0.62 respectively). Their study identified 25 radiomic features significantly related to response to neoadjuvant chemotherapy including GLCM and GLRLM, with more than half of the features belonging to the latter group [30]. Our study found ten radiomic features (five for each analysis performed); among these features, GLCM and GLRLM showed interesting results. GLCM and GLRLM are features expressing the signal heterogeneity within a lesion. They represent the relative relationship between the distribution and location of the gray level. In general, these features (GLCM and GLRLM) are more pronounced in patients with lack of response to neoadjuvant chemotherapy. They are possible expressions of the more pronounced intra-tumoral heterogeneity in non-responders than responders. The Cluster Shade represents a measure of the skewness and uniformity of the GLCM, and Autocorrelation expresses the magnitude of the fineness or coarseness of a lesion's texture. Although our results regarding Cluster Shade are in line with this trend, Autocorrelation showed the opposite trend and further investigation is needed to explore this. Among GLRLM features, LongRunEmphasis, a measure of the distribution of long run lengths, showed greater value, longer run lengths, and more coarse structural textures. Our $\Delta$ Radiomics for this feature showed that responders had a wider delta compared to non-responders. This is explicable with a reduced LongRunEmphasis after $\mathrm{p}-\mathrm{ChT}$ in responders. As already reported in many studies conducted on different tumor types, the heterogeneity of radiomics features is greater in more aggressive tumors in terms of proliferation, angiogenesis, and metastatic spread. These characteristics have often resulted in chemotherapy resistance [31,32].

The $\Delta$ Radiomics data of our study are also in line with a study performed by Mazzei et al. [33]. The study authors analyzed $\Delta$ Radiomics on pre-ChT in 23 advanced gastric cancer patients. On multivariate analysis, their results showed a significant correlation between the delta GLCM Contrast features and complete pathological response. Additionally, our analysis showed how Contrast features have good diagnostic ability in differentiating responders from non-responders.

Despite the interesting results, our study has some limitations, as follows: (a) the small cohort of patients made it impossible to perform training and validation for sub-groups and led to possible instability of the results; (b) the lack of multivariate analysis with clinical and radiological findings; (c) the lack of correlation with histologic and molecular expressions of the tumor; (d) the lack of radiomics analysis on the arterial phase; (e) the lack of different iterative reconstruction levels applied to $\mathrm{CT}$ acquisition that might alter radiomic features (we applied fixed iterative reconstruction percentages to avoid bias since 
this aspect was beyond the aim of the study); and finally (f) the manual segmentation of the whole gastric tumor.

\section{Conclusions}

Our preliminary results suggest the potential role of radiomics in the assessment of response to perioperative chemotherapy in gastric cancer patients. This new non-invasive imaging biomarker could be integrated into the comprehensive evaluation of gastric cancer patients to provide an early assessment of patient response to chemotherapy and provide improved therapeutic management. However, further investigations are needed to confirm the data before they are given full consideration in the clinical setting.

Author Contributions: Conceptualization, G.M.G., M.Z., D.C. and A.L.; methodology, G.M.G. and D.C; software, M.Z. and E.B.; validation, F.M., E.P. and G.G.L.; formal analysis, M.Z., E.B. and D.C.; investigation, M.P., G.G. and T.P.; resources, E.P. and B.A.; data curation, P.M., M.T., F.M. and G.G.L.; writing-original draft preparation, M.Z, M.T. and C.R.; writing-review and editing, G.M.G., E.I., P.M. and D.C; visualization, P.M. and G.G.L.; supervision, A.L., B.A. and D.C.; project administration, B.A. and A.L. All authors have read and agreed to the published version of the manuscript.

Funding: This research received no external funding.

Institutional Review Board Statement: The study was conducted according to the guidelines of the Declaration of Helsinki. Formal Institutional Review Board approval was not required because of the non-interventional retrospective design; however, signed consent for the treatment and the analysis of data for scientific purposes was obtained from all patients before any procedure was performed.

Informed Consent Statement: Informed consent was obtained from all subjects involved in the study. Written informed consent has been obtained from the patient(s) to publish this paper.

Data Availability Statement: Data supporting results can be provided by the corresponding author Damiano Caruso.

Conflicts of Interest: The authors declare no conflict of interest.

\section{References}

1. Ferlay, J.; Steliarova-Foucher, E.; Lortet-Tieulent, J.; Rosso, S.; Coebergh, J.W.; Comber, H.; Forman, D.; Bray, F. Cancer incidence and mortality patterns in Europe: Estimates for 40 countries in 2012. Eur. J. Cancer 2013, 49, 1374-1403. [CrossRef]

2. Bray, F.; Ferlay, J.; Soerjomataram, I.; Siegel, R.L.; Torre, L.A.; Jemal, A. Global cancer statistics 2018: GLOBOCAN estimates of incidence and mortality worldwide for 36 cancers in 185 countries. CA: A Cancer J. Clin. 2018, 68, 394-424. [CrossRef]

3. Japanese Gastric Cancer Association. Japanese gastric cancer treatment guidelines 2014 (ver. 4). Gastric Cancer 2017, $20,1-19$. [CrossRef]

4. Smyth, E.C.; Verheij, M.; Allum, W.; Cunningham, D.; Cervantes, A.; Arnold, D.; Committee, E.G. Gastric cancer: ESMO Clinical Practice Guidelines for diagnosis, treatment and follow-up. Ann. Oncol. 2016, 27, v38-v49. [CrossRef] [PubMed]

5. Cunningham, D.; Allum, W.H.; Stenning, S.P.; Thompson, J.N.; Van de Velde, C.J.; Nicolson, M.; Scarffe, J.H.; Lofts, F.J.; Falk, S.J.; Iveson, T.J.; et al. Perioperative chemotherapy versus surgery alone for resectable gastroesophageal cancer. N. Engl. J. Med. 2006, 355, 11-20. [CrossRef] [PubMed]

6. Thuss, P.; Wolfgang, F.; Jorg, T.; Michael, K.; Claudia, P.; Thorsten Oliver, G.; Elke, J.; Johannes, M.; Martin, H.S.; Ralf Hofheinz, S.-E.A.-B.; et al. Perioperative chemotherapy with docetaxel, oxaliplatin, and fluorouracil/leucovorin (FLOT) versus epirubicin, cisplatin, and fluorouracil or capecitabine (ECF/ECX) for resectable gastric or gastroesophageal junction (GEJ) adenocarcinoma (FLOT4-AIO): A multicenter, randomized phase 3 trial. J. Clin. Oncol. 2017. [CrossRef]

7. Smyth, E.C.; Wotherspoon, A.; Peckitt, C.; Gonzalez, D.; Hulkki-Wilson, S.; Eltahir, Z.; Fassan, M.; Rugge, M.; Valeri, N.; Okines, A.; et al. Mismatch Repair Deficiency, Microsatellite Instability, and Survival: An Exploratory Analysis of the Medical Research Council Adjuvant Gastric Infusional Chemotherapy (MAGIC) Trial. JAMA Oncol. 2017, 3, 1197-1203. [CrossRef]

8. Petrillo, A.; Pompella, L.; Tirino, G.; Pappalardo, A.; Laterza, M.M.; Caterino, M.; Orditura, M.; Ciardiello, F.; Lieto, E.; Galizia, G.; et al. Perioperative Treatment in Resectable Gastric Cancer: Current Perspectives and Future Directions. Cancers 2019, 11, 399. [CrossRef] [PubMed]

9. Kohlruss, M.; Grosser, B.; Krenauer, M.; Slotta-Huspenina, J.; Jesinghaus, M.; Blank, S.; Novotny, A.; Reiche, M.; Schmidt, T.; Ismani, L.; et al. Prognostic implication of molecular subtypes and response to neoadjuvant chemotherapy in 760 gastric carcinomas: Role of Epstein-Barr virus infection and high- and low-microsatellite instability. J. Pathol. Clin. Res. 2019, 5, 227-239. [CrossRef] [PubMed] 
10. De Cecco, C.N.; Ganeshan, B.; Ciolina, M.; Rengo, M.; Meinel, F.G.; Musio, D.; De Felice, F.; Raffetto, N.; Tombolini, V.; Laghi, A. Texture analysis as imaging biomarker of tumoral response to neoadjuvant chemoradiotherapy in rectal cancer patients studied with 3-T magnetic resonance. Investig. Radiol. 2015, 50, 239-245. [CrossRef]

11. Yip, S.S.; Aerts, H.J. Applications and limitations of radiomics. Phys. Med. Biol. 2016, 61, R150-R166. [CrossRef]

12. Ferrari, R.; Mancini-Terracciano, C.; Voena, C.; Rengo, M.; Zerunian, M.; Ciardiello, A.; Grasso, S.; Mare, V.; Paramatti, R.; Russomando, A.; et al. MR-based artificial intelligence model to assess response to therapy in locally advanced rectal cancer. Eur. J. Radiol. 2019, 118, 1-9. [CrossRef] [PubMed]

13. Caruso, D.; Zerunian, M.; Ciolina, M.; de Santis, D.; Rengo, M.; Soomro, M.H.; Giunta, G.; Conforto, S.; Schmid, M.; Neri, E.; et al. Haralick's texture features for the prediction of response to therapy in colorectal cancer: A preliminary study. Radiol. Med. 2018, 123, 161-167. [CrossRef] [PubMed]

14. Caruso, D.; Polici, M.; Zerunian, M.; Pucciarelli, F.; Guido, G.; Polidori, T.; Landolfi, F.; Nicolai, M.; Lucertini, E.; Tarallo, M.; et al. Radiomics in Oncology, Part 1: Technical Principles and Gastrointestinal Application in CT and MRI. Cancers 2021, 13, 2522. [CrossRef] [PubMed]

15. Caruso, D.; Polici, M.; Zerunian, M.; Pucciarelli, F.; Guido, G.; Polidori, T.; Landolfi, F.; Nicolai, M.; Lucertini, E.; Tarallo, M.; et al. Radiomics in Oncology, Part 2: Thoracic, Genito-Urinary, Breast, Neurological, Hematologic and Musculoskeletal Applications. Cancers 2021, 13, 2681. [CrossRef]

16. Giganti, F.; Antunes, S.; Salerno, A.; Ambrosi, A.; Marra, P.; Nicoletti, R.; Orsenigo, E.; Chiari, D.; Albarello, L.; Staudacher, C.; et al. Gastric cancer: Texture analysis from multidetector computed tomography as a potential preoperative prognostic biomarker. Eur. Radiol. 2017, 27, 1831-1839. [CrossRef] [PubMed]

17. Li, Z.; Zhang, D.; Dai, Y.; Dong, J.; Wu, L.; Li, Y.; Cheng, Z.; Ding, Y.; Liu, Z. Computed tomography-based radiomics for prediction of neoadjuvant chemotherapy outcomes in locally advanced gastric cancer: A pilot study. Chin. J. Cancer Res. 2018, 30, 406-414. [CrossRef]

18. Giganti, F.; Marra, P.; Ambrosi, A.; Salerno, A.; Antunes, S.; Chiari, D.; Orsenigo, E.; Esposito, A.; Mazza, E.; Albarello, L.; et al. Pre-treatment MDCT-based texture analysis for therapy response prediction in gastric cancer: Comparison with tumour regression grade at final histology. Eur. J. Radiol. 2017, 90, 129-137. [CrossRef]

19. Becker, K.; Mueller, J.D.; Schulmacher, C.; Ott, K.; Fink, U.; Busch, R.; Bottcher, K.; Siewert, J.R.; Hofler, H. Histomorphology and grading of regression in gastric carcinoma treated with neoadjuvant chemotherapy. Cancer 2003, 98, 1521-1530. [CrossRef]

20. Amin, M.B.; Greene, F.L.; Edge, S.B.; Compton, C.C.; Gershenwald, J.E.; Brookland, R.K.; Meyer, L.; Gress, D.M.; Byrd, D.R.; Winchester, D.P. The Eighth Edition AJCC Cancer Staging Manual: Continuing to build a bridge from a population-based to a more "personalized" approach to cancer staging. CA Cancer J. Clin. 2017, 67, 93-99. [CrossRef]

21. AJCC-AJCC 8th Edition Cancer Staging Form, Histology and Topography Supplements Available Now. Available online: https:/ / cancerstaging.org/About/news/Pages / AJCC-8th-Edition-Cancer-Staging-Form-and-Histology-and-TopographySupplements-Available-Now.aspx (accessed on 28 March 2021).

22. Caruso, D.; Rosati, E.; Panvini, N.; Rengo, M.; Bellini, D.; Moltoni, G.; Bracci, B.; Lucertini, E.; Zerunian, M.; Polici, M.; et al. Optimization of contrast medium volume for abdominal CT in oncologic patients: Prospective comparison between fixed and lean body weight-adapted dosing protocols. Insights Imaging 2021, 12, 40. [CrossRef]

23. Caruso, D.; De Santis, D.; Rivosecchi, F.; Zerunian, M.; Panvini, N.; Montesano, M.; Biondi, T.; Bellini, D.; Rengo, M.; Laghi, A. Lean Body Weight-Tailored Iodinated Contrast Injection in Obese Patient: Boer versus James Formula. Biomed. Res. Int. 2018, 2018, 8521893. [CrossRef] [PubMed]

24. Caruso, D.; Zerunian, M.; Pucciarelli, F.; Bracci, B.; Polici, M.; D’Arrigo, B.; Polidori, T.; Guido, G.; Barbato, L.; Polverari, D.; et al. Influence of Adaptive Statistical Iterative Reconstructions on CT Radiomic Features in Oncologic Patients. Diagnostics 2021, 11, 1000. [CrossRef]

25. Ma, Z.; Fang, M.; Huang, Y.; He, L.; Chen, X.; Liang, C.; Huang, X.; Cheng, Z.; Dong, D.; Xie, J.; et al. CT-based radiomics signature for differentiating Borrmann type IV gastric cancer from primary gastric lymphoma. Eur. J. Radiol. 2017, 91, 142-147. [CrossRef] [PubMed]

26. van Griethuysen, J.J.M.; Fedorov, A.; Parmar, C.; Hosny, A.; Aucoin, N.; Narayan, V.; Beets-Tan, R.G.H.; Fillion-Robin, J.C.; Pieper, S.; Aerts, H. Computational Radiomics System to Decode the Radiographic Phenotype. Cancer Res. 2017, 77, e104-e107. [CrossRef]

27. Neves Filho, E.H.; de Sant'Ana, R.O.; Nunes, L.V.; Pires, A.P.; da Cunha, M.D. Histopathological regression of gastric adenocarcinoma after neoadjuvant therapy: A critical review. APMIS 2017, 125, 79-84. [CrossRef]

28. Liu, S.; Liu, S.; Ji, C.; Zheng, H.; Pan, X.; Zhang, Y.; Guan, W.; Chen, L.; Guan, Y.; Li, W.; et al. Application of CT texture analysis in predicting histopathological characteristics of gastric cancers. Eur. Radiol. 2017, 27, 4951-4959. [CrossRef] [PubMed]

29. Wang, Y.; Liu, W.; Yu, Y.; Liu, J.J.; Xue, H.D.; Qi, Y.F.; Lei, J.; Yu, J.C.; Jin, Z.Y. CT radiomics nomogram for the preoperative prediction of lymph node metastasis in gastric cancer. Eur. Radiol. 2020, 30, 976-986. [CrossRef]

30. Sun, K.Y.; Hu, H.T.; Chen, S.L.; Ye, J.N.; Li, G.H.; Chen, L.D.; Peng, J.J.; Feng, S.T.; Yuan, Y.J.; Hou, X.; et al. CT-based radiomics scores predict response to neoadjuvant chemotherapy and survival in patients with gastric cancer. BMC Cancer 2020, 20, 468. [CrossRef] [PubMed] 
31. Braman, N.M.; Etesami, M.; Prasanna, P.; Dubchuk, C.; Gilmore, H.; Tiwari, P.; Plecha, D.; Madabhushi, A. Intratumoral and peritumoral radiomics for the pretreatment prediction of pathological complete response to neoadjuvant chemotherapy based on breast DCE-MRI. Breast Cancer Res. 2017, 19, 57. [CrossRef] [PubMed]

32. Ganeshan, B.; Goh, V.; Mandeville, H.C.; Ng, Q.S.; Hoskin, P.J.; Miles, K.A. Non-small cell lung cancer: Histopathologic correlates for texture parameters at CT. Radiology 2013, 266, 326-336. [CrossRef] [PubMed]

33. Mazzei, M.A.; Nardone, V.; Di Giacomo, L.; Bagnacci, G.; Gentili, F.; Tini, P.; Marrelli, D.; Volterrani, L. The role of delta radiomics in gastric cancer. Quant. Imaging Med. Surg. 2018, 8, 719-721. [CrossRef] [PubMed] 